\title{
InP-on-silicon electronically tunable lasers
}

\author{
Sören Dhoore*, Gunther Roelkens, Geert Morthier \\ * Photonics Research Group, INTEC, Ghent University - imec, Ghent, Belgium \\ e-mail: Soren.Dhoore@UGent.be
}

\begin{abstract}
In this paper we present our recent results on heterogeneously integrated InP-on-silicon tunable lasers. DBR-, DFB- and AWG-based laser structures on SOI are discussed. Emphasis is laid on electronically tunable laser types, which can allow for (sub-) nanosecond fast wavelength tuning. The discussed laser devices are expected to find application in future advanced optical networks for intra- and inter-data center communication.
\end{abstract}

Keywords: tunable lasers, fast wavelength tuning, heterogeneous integration, silicon photonics.

\section{INTRODUCTION}

Since the advent of the World Wide Web in the early nineties, Internet technologies have known an incredibly fast development. Cloud computing and other web applications have led to an enormous increase in the amount of data being retrieved from and processed by data centers [1]. In order to sustain the increasing demand for bandwidth, response speed and data storage, more powerful data centers will be required, with very fast optical interconnects between the employed servers. The packet or burst nature of traffic in these data centers has renewed the interest in flexible interconnect architectures using optical packet switching [2]. Typical implementations of optical packet switches or cross connects are based on fixed routers and reconfigurable line cards making use of fast tunable lasers with nanosecond switching speeds [3]. This means that there is going to be a strong demand for very high speed and very fast tunable optical transmitters with high power efficiency [4]. Nowadays VCSELs are still the preferred light sources for short distance interconnects, but don't give enough optical power for distances beyond a few hundred meters nor do they allow fast wavelength switching. InP-onsilicon edge-emitting lasers such as DFBs have demonstrated large optical output powers with high modulation bandwidth and are hence favored for longer distances. Moreover, the output of such InP-on-silicon lasers is coupled to silicon-on-insulator (SOI) waveguides, which makes the laser devices excellent candidates for integration with other silicon-photonics-based passive waveguide components. So far the research on tunable InP-on-silicon lasers has mainly been directed towards thermally tunable lasers since these types typically yield a narrow emission linewidth and thus small phase noise, a feature which is desired for coherent optical communication. While such lasers have already been demonstrated, their tuning speed is too low to find application in optical packet switching. Electronically tunable lasers on the other hand do allow fast tuning but are more difficult to implement on SOI. One of the reasons is that the free-carrier plasma dispersion effect used to realize fast electronic tuning is about a factor 10 weaker for silicon than for typical InP compounds. In this paper, we give an overview of our recent research efforts to realize InP-on-silicon electronically tunable lasers. Three laser types are discussed. Some of the lasers suffer from parasitic self-heating effects although with our most recent devices fast wavelength tuning is achieved. Further optimization of the laser structure can enhance the laser threshold and overall wavelength tuning range, which could make the devices attractive candidates for the aforementioned applications in advanced optical networks.

\section{INP-ON-SILICON THREE-SECTION DBR LASER}

\subsection{Laser structure}

A 3D schematic of the first laser is shown in Fig. 1(a) [5]. The laser consists of a passive SOI waveguide circuit on which an InP/InGaAsP TTG membrane [6] is integrated by means of adhesive DVS-BCB bonding [7]. The TTG membrane contains an InGaAsP double heterojunction active multiple quantum well (MQW) and a passive tuning layer in which carriers can be injected to provide optical gain and phase tuning, respectively. A 100-nmthick n-InP separation layer electronically decouples both regions and serves as the common ground contact layer. The laser cavity is formed by a broadband reflecting facet (reflectance $~ 30 \%$ ) and a highly reflective DBR mirror (peak reflectance $>90 \%$ ). The latter is implemented as a first-order sidewall-corrugated waveguide grating defined in the SOI device layer. The SOI waveguide structures are fabricated in a CMOS pilot-line at imec through deep UV lithography and have a 400-nm-thick silicon device layer with an etch depth of $180 \mathrm{~nm}$. In the presented laser structure, three longitudinal sections can be discerned: a gain, a phase, and a Bragg section. In the gain section, current $\left(I_{\text {gain }}\right)$ is injected into the active layer to provide optical gain. The tuning layer is left unbiased. In the phase and Bragg sections, the active layer is removed during fabrication, and current ( $\mathrm{I}_{\text {phase }}$ and $\mathrm{I}_{\mathrm{Bragg}}$, respectively) is injected into the tuning layer. Upon current injection, the effective index of the waveguide 

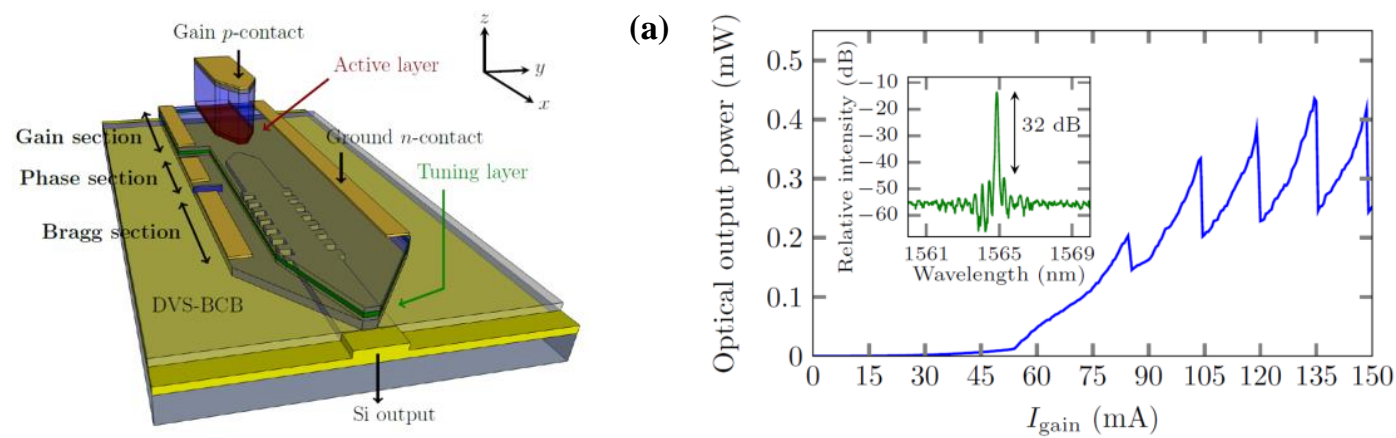

(b)

Figure 1. (a) 3D schematic of the three-section DBR laser structure; (b) LI characteristic.

mode is modified through the free-carrier plasma dispersion effect and Joule heating. Note that both effects are counteracting and lead to a blue and a red shift of the Bragg wavelength, respectively.

\subsection{Characterization results}

Figure 1(b) shows the light-current (LI) characteristic for the DBR laser when the phase and Bragg sections are left unbiased. The threshold current is $55 \mathrm{~mA}$ and the maximum CW on-chip optical output power is $0.4 \mathrm{~mW}$. The peaks in the LI characteristic are due to longitudinal mode hopping. The inset of Fig. 1(b) shows the optical spectrum, measured at $\mathrm{I}_{\text {gain }}=120 \mathrm{~mA}$. Single-mode lasing operation is achieved at $1565 \mathrm{~nm}$ with a SMSR of $32 \mathrm{~dB}$. The free spectral range (FSR) of the laser cavity is $0.5 \mathrm{~nm}$. Figure 2(a) shows the lasing wavelength as a function of $I_{\text {Bragg }}$ when $I_{\text {phase }}$ is varied between 0 and $24 \mathrm{~mA}$. Clearly all wavelengths between $1565 \mathrm{~nm}$ and $1577 \mathrm{~nm}$ are accessible, and continuous tuning over the $12 \mathrm{~nm}$ wavelength range can be achieved through simultaneous adjustment of $\mathrm{I}_{\text {phase }}$ and $\mathrm{I}_{\text {Bragg. }}$. Figure 2(b) shows the superimposed lasing spectra across the tuning range. The SMSR remains larger than $30 \mathrm{~dB}$ and the optical output power varies only within $2 \mathrm{~dB}$ over the entire tuning range. The red shift of the lasing wavelength with increasing tuning current indicates a dominant heating effect. Simulations indicate that through a modified epitaxial layer stack design (e.g., with a thicker tuning and surrounding cladding layer) the optical confinement factor in the tuning layer can be significantly increased, resulting in a stronger electronic effect and hence faster tuning $(\sim \mathrm{ns})$.

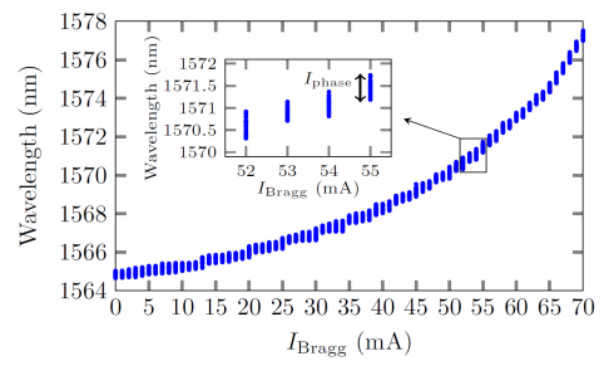

(a)

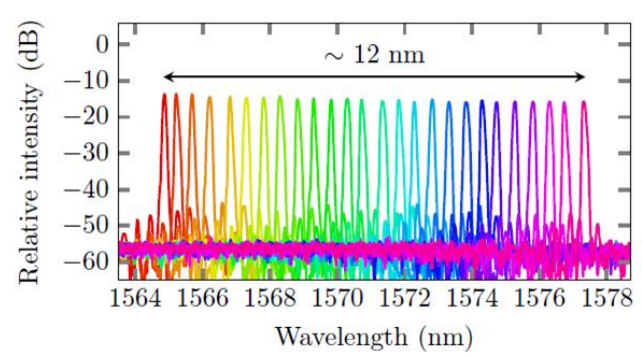

(b)

Figure 2. (a) Lasing wavelength vs $I_{\text {phase }}$ and $I_{\text {Bragg; }}$ (b) Superimposed lasing spectra.

\section{INP-ON-SILICON TUNABLE DFB LASER}

The second laser type is conceptually similar to the first laser type but constitutes a DFB-laser type. A crosssection is shown in Fig. 3(a). Again use is made of a TTG membrane. This time a rather thick tuning layer of $200 \mathrm{~nm}$ is used to have a large optical confinement in the tuning layer. The mode profile is shown in the inset of Fig. 3(a). Preliminary measurement results indicate a threshold current of $45 \mathrm{~mA}$ at room temperature, with a maximum fiber-coupled output power of $0.1 \mathrm{~mW}$ for a laser device with a grating length of $600 \mu \mathrm{m}$. Tuning characteristics are presented in Fig. 3(b), which shows the superimposed lasing spectra at different tuning currents. The tuning range is limited to about $2.5 \mathrm{~nm}$, which already allows to address 4 wavelength channels on a $0.8 \mathrm{~nm}$ dense wavelength-division multiplexing (DWDM) grid. The blue shift of the lasing wavelength now indicates a net electronic tuning effect. A new fabrication run is on the way to further improve the laser and tuning characteristics.

\section{INP-ON-SILICON AWG-BASED FILTERED FEEDBACK TUNABLE LASER}

\subsection{Laser structure}

The third and final laser structure is conceptually rather different from the previously discussed laser types. A schematic is shown in Fig. 4(a) [8]. The laser consists of a main Fabry-Pérot (FP) cavity with two partially reflective broadband SOI DBR mirrors (denoted as $\mathrm{DBR}_{\mathrm{FF}}$ and $\mathrm{DBR}_{\text {Out }}$ respectively). At one side of the FP 


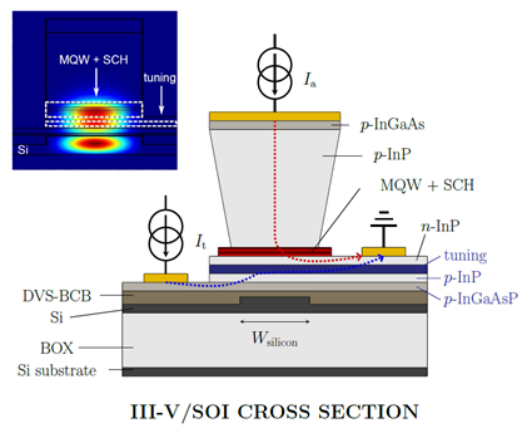

(a)

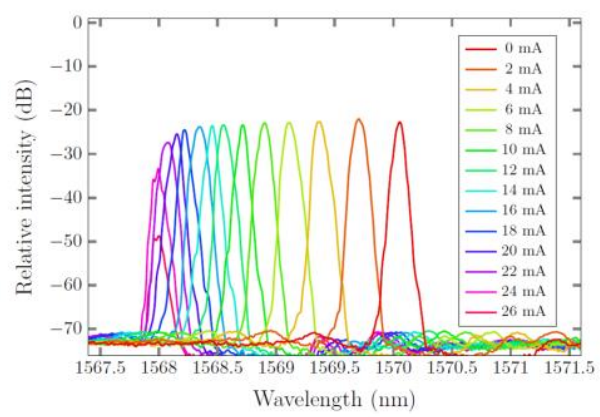

(b)

Figure 3. (a) Cross-section of the tunable DFB laser structure; (b) Superimposed lasing spectra.

cavity the light output is coupled to an SOI arrayed waveguide grating (AWG) filter that demultiplexes the light in four different waveguide branches. In each branch an InP-on-silicon semiconductor optical amplifier (SOA) is implemented that can function as optical gate. If the SOA is biased at or above transparency, input light can be efficiently transmitted or even be amplified. When unbiased, all incident light is absorbed in the gate. By making use of a highly reflective SOI DBR mirror at the end of each waveguide branch (denoted as DBR $\mathrm{HR}_{\text {) }}$ ) light can be fed back to the laser. This enables the laser to operate in single-mode regime, close to the wavelength with maximum transmission through the AWG filter [9]. The filtered feedback principle is used to achieve singlemode laser operation and tunability. InP-on-silicon lasers making use of AWGs have been demonstrated before, where the AWG was an integral part of the laser cavity [10]. Because of their long cavity length, such lasers usually have a low modulation bandwidth. In our implementation the AWG is not part of the main cavity. The filtered feedback concept was first introduced and experimentally demonstrated by B. Docter et al. [11], where discrete wavelength tuning was achieved with a pure InP-based laser. The implementation on silicon, however, allows to benefit from the superior passive functionality of silicon, leading to improved static and dynamic lasing characteristics.

\subsection{Static characterization results}

The lasing spectrum at room temperature for $\mathrm{I}_{\mathrm{G} a i n}=80 \mathrm{~mA}$ with the SOA gates biased at or above transparency is shown in Fig. 4(b). Single-mode laser operation is achieved with a SMSR larger than $35 \mathrm{~dB}$ for all four wavelength channels. The channel spacing is $4 \mathrm{~nm}$. CW laser operation up to $65{ }^{\circ} \mathrm{C}$ is achieved. At room temperature the laser threshold is $20 \mathrm{~mA}$, with a maximum fiber-coupled output power of $0.1 \mathrm{~mW}$, which corresponds to a waveguide-coupled output power of $4 \mathrm{~mW}$.

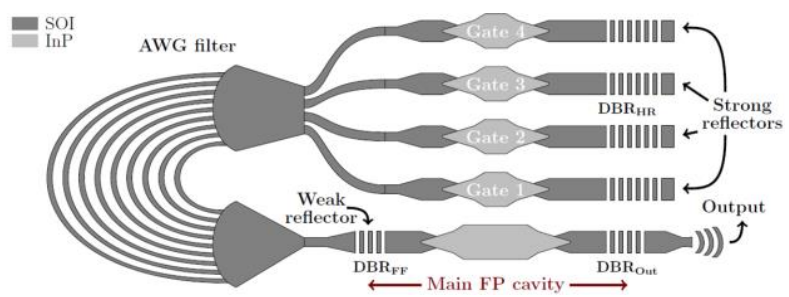

(a)

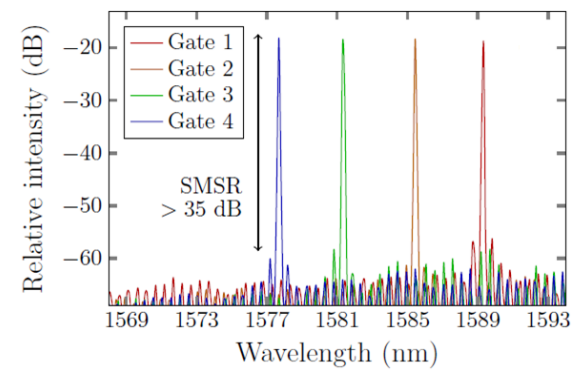

(b)

Figure 4. (a) Schematic of the AWG-based filtered feedback tunable laser structure; (b) Lasing spectra.
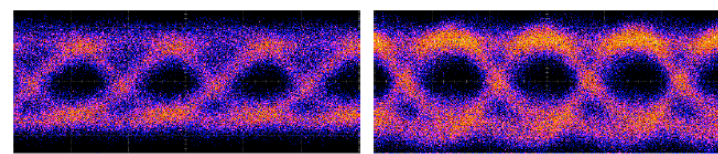

Channel $1-12.5 \mathrm{Gbit} / \mathrm{s}$

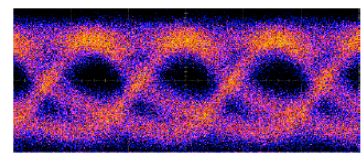

Channel 2 - 12.5 Gbit/s

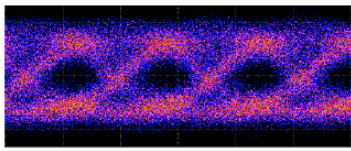

Channel $3-12.5 \mathrm{Gbit} / \mathrm{s}$ Channel $4-12.5 \mathrm{Gbit} / \mathrm{s}$ (a)

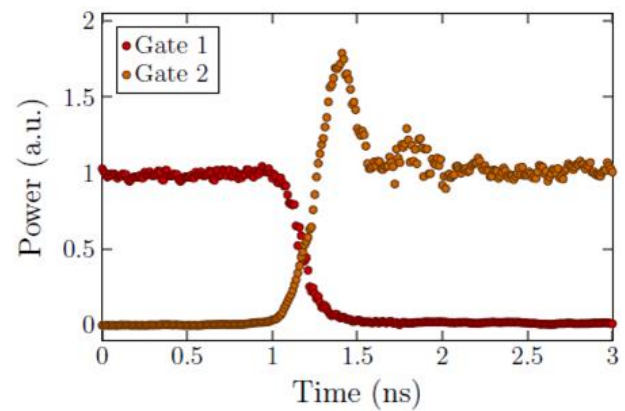

(b)

Figure 5. (a) Eye diagrams for the filtered-feedback tunable laser; (b) Wavelength switching characteristics. 


\subsection{Dynamic characterization results}

\subsubsection{Large-signal modulation}

For the large-signal characterization of the laser a large-amplitude non-return-to-zero on-off-keying (NRZ-OOK) electrical signal is generated at different bitrates. A $3 \mathrm{Vpp}$ RF voltage swing is applied to the laser device with an $\mathrm{I}_{\mathrm{Gain}}=80 \mathrm{~mA}$ DC bias. Eye diagrams are measured by means of a $40 \mathrm{GHz}$ photodiode of which the output is connected to a real-time oscilloscope. Open eye diagrams are obtained up to $12.5 \mathrm{Gbit} / \mathrm{s}$ for the four different wavelength channels. This is shown in Fig. 5(a). A bit-error rate (BER) below $3.8 \cdot 10^{-3}$ can be obtained, which enables error-free operation assuming 7\% overhead hard decision forward error correction (HD-FEC).

\subsubsection{Wavelength switching}

As proof-of-principle wavelength tuning is done by periodically switching the bias between Gate 1 and Gate 2 with a $500 \mathrm{~ns}$ periodic rectangular $0 \mathrm{~mA}-50 \mathrm{~mA}$ pulse train. The wavelength switching characteristics are shown in Fig. 5(b). Clearly, wavelength switching occurs in less than a nanosecond with a rise time of 195 ps. The fall time is 190 ps. Hereby rise (fall) time is defined as the time it takes for the response to rise (fall) from $10 \%(90 \%)$ to $90 \%(10 \%)$ of the steady-state response.

\section{CONCLUSION}

Three InP-on-silicon electronically tunable laser types have been discussed. The first DBR-like structure allows for $12 \mathrm{~nm}$ continuous, albeit thermal tuning through current injection in the tuning layer of a TTG membrane. The second laser structure is a TTG-based DFB laser for which electronic tuning is achieved with a limited tuning range of $2.5 \mathrm{~nm}$. The final AWG-based laser structure enables discrete wavelength tuning through optical filtered feedback and has the advantage of extremely fast wavelength tuning (sub-nanosecond) and does not make use of the rather complex TTG epitaxial layer stack. Current research efforts try to merge the best of both to realize nanosecond fast wavelength tunable lasers with large tuning ranges and good static and dynamic laser characteristics.

\section{ACKNOWLEDGEMENTS}

The authors acknowledge the Methusalem programme of the Flemish government for funding.

\section{REFERENCES}

[1] Q. Zhang, L. Cheng, R. Boutaba, “Cloud computing: state-of-the-art and research challenges," J. of Internet Serv. and Appl. 1(1), 7-18 (2010).

[2] C. Kachris, K. Kanonakis, and I. Tomkos, "Optical interconnection networks in data centers: recent trends and future challenges,” IEEE Commun. Mag. (2013).

[3] Q. Huang, Y.-K. Yeo, and L. Zhou, “A single-stage optical load-balanced switch for data centers," Opt. Express 20(22), 25014-25021 (2012).

[4] Y. Wen, H. Jin, M. Chen, V. C. M. Leung, "Enabling Technologies for Future Data Center Networking: A Primer," IEEE Netw. 27(4), 8-15 (2013).

[5] S. Dhoore, G. Roelkens, and G. Morthier, "III-V-on-silicon three-section DBR laser with over $12 \mathrm{~nm}$ continuous tuning range," Opt. Lett. 42(6), 1121-1124 (2017).

[6] M. C. Amann, S. Illek, C. Schanen, and W. Thulke, "Tunable twin-guide laser: A novel laser diode with improved tuning performance," Applied Physics Letters, 54(25), 2532-2533 (1989).

[7] G. Roelkens, A. Abbasi, P. Cardile, U. Dave, A. De Groote, Y. De Koninck, S. Dhoore, X. Fu, A. Gassenq, N. Hattasan, Q. Huang, S. Kumari, S. Keyvaninia, B. Kuyken, L. Li, P. Mechet, M. Muneeb, D. Sanchez, H. Shao, T. Spuesens, A. Z. Subramanian, S. Uvin, M. Tassaert, K. Van Gasse, J. Verbist, R. Wang, Z. Wang, J. Zhang, J. Van Campenhout, X. Yin, J. Bauwelinck, G. Morthier, R. Baets, and D. Van Thourhout, "III-V-on-silicon photonic devices for optical communication and sensing," Photonics, 2(3), 969-1004 (2015).

[8] S. Dhoore, A. Rahim, G. Roelkens, G. Morthier, "12.5 Gbit/s discretely tunable InP-on-silicon filtered feedback laser with sub-nanosecond wavelength switching times," Opt. Express 26(7), 8059-8068 (2018).

[9] D. M. Kane, and K. A. Shore, "Unlocking dynamical diversity: optical feedback effects on semiconductor lasers," Wiley (2005).

[10] G. Kurczveil, J. M. Heck, J. D. Peters, J. M. Garcia, D. Spencer, and J. E. Bowers, “An integrated hybrid silicon multiwavelength AWG laser,” IEEE J. Sel. Topics Quantum Electron. 17(6), 1521-1527 (2011).

[11] B. Docter, J. Pozo, S. Beri, I. V. Ermakov, J. Danckaert, M. K. Smit, and F. Karouta, "Discretely tunable laser based on filtered feedback for telecommunication applications," IEEE J. Sel. Topics Quantum Electron. 16(5), 1405-1412 (2010). 